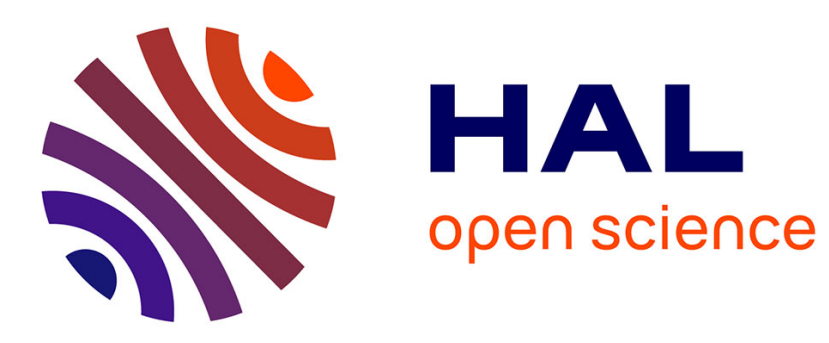

\title{
Theoretical modelling of S-band thulium-doped silica fibre amplifiers
}

Pavel Peterka, Basile Faure, Wilfried Blanc, M. Karasek, Bernard Dussardier

\section{To cite this version:}

Pavel Peterka, Basile Faure, Wilfried Blanc, M. Karasek, Bernard Dussardier. Theoretical modelling of S-band thulium-doped silica fibre amplifiers. Optical and Quantum Electronics, 2004, 36, pp.201212. 10.1023/B:OQEL.0000015640.82309.7d . hal-00732467

\section{HAL Id: hal-00732467 https://hal.science/hal-00732467}

Submitted on 14 Sep 2012

HAL is a multi-disciplinary open access archive for the deposit and dissemination of scientific research documents, whether they are published or not. The documents may come from teaching and research institutions in France or abroad, or from public or private research centers.
L'archive ouverte pluridisciplinaire HAL, est destinée au dépôt et à la diffusion de documents scientifiques de niveau recherche, publiés ou non, émanant des établissements d'enseignement et de recherche français ou étrangers, des laboratoires publics ou privés. 


\title{
Theoretical modelling of S-band thulium-doped silica fibre amplifiers
}

\author{
P. Peterka ${ }^{2,1, *}$, B. Faure ${ }^{1}$, W. Blanc ${ }^{1}$, M. Karásek ${ }^{2}$ and B. Dussardier ${ }^{1}$ \\ ${ }^{1}$ Laboratoire de Physique de la Matière Condensée CNRS, Université de Nice - \\ Sophia Antipolis, Parc Valrose, 06108 Nice, France \\ ${ }^{2}$ Institute of Radio Engineering and Electronics AS CR, 18251 Praha 8, Czech \\ Republic \\ (*author for correspondence: E-mail: pavel.peterka@ure.cas.cz)
}

May 27, 2003

\begin{abstract}
A comprehensive numerical model of a thulium-doped silica-based fibre amplifiers is presented. The model is spectrally and spatially resolved and is general in terms of pumping scheme used. The application of the model for predicting the S-band amplifier performance and for optimization of amplifier parameters is shown. For optimized Tm-doped fibre with ${ }^{3} H_{4}$ level lifetime of $45 \mu \mathrm{s}$, which is the maximum value in the Tm-doped silica fibres prepared by the authors, above $20 \mathrm{~dB}$ of gain with $2000 \mathrm{~mW}$ pump power at the $1050 \mathrm{~nm}$ pump band can be expected according to the simulations.
\end{abstract}

Keywords: fibre amplifiers, fibre lasers, numerical modelling, thulium, optical fibres

\section{Introduction}

Increasing demand for the transmission capacity in telecommunications, driven mainly by the Internet, stimulates development of optical communication systems with larger bandwidth. Current long-haul transmission systems use erbium doped fibre amplifiers, EDFA's, that operate in the so-called C- and L-band (1530-1565 nm and 1570-1610 $\mathrm{nm}$, respectively). These two bands represent only a smaller portion of low-loss spectral region available in standard single-mode fibres (1440$1650 \mathrm{~nm}$ ) or newly deployed fibres with low $\mathrm{OH}^{-}$absorption (1300$1650 \mathrm{~nm}$ ). The next logical frontier following the C- and L-bands is the S-band (1460-1530 $\mathrm{nm}$ ) and among the most promising candidates for optical amplification in this spectral region are the thulium-doped fibre amplifiers, TDFA's.

In thulium the stimulated emission at wavelengths around $1.47 \mu \mathrm{m}$ occurs between the two excited levels ${ }^{3} H_{4} \rightarrow{ }^{3} F_{4}$, as was demonstrated for the first time already by Antipenko et al. (1983). Two main problems complicate the use of this transition for an efficient optical amplification. Firstly, the thulium behaves as a four-level laser system where the lifetime of the upper level is shorter than that of the lower level. Therefore the population inversion can be hardly achieved with 
direct pumping to the upper laser level. Komukai et al. (1995) have solved this problem in Tm-doped fluoride fibre, using an upconversion pumping by Nd:YAG laser at $1064 \mathrm{~nm}$, see the utmost left scheme in Fig. 1. In this pumping scheme, the Tm ions deexcited to the ${ }^{3} F_{4}$ level can be immediately pumped back to the upper laser level ${ }^{3} \mathrm{H}_{4}$. In order to achieve higher power conversion efficiency (PCE) of the amplifier or to shape its spectral gain profile, another single-wavelength and various dual-wavelengths pumping schemes were reported, which are also shown in Fig. 1.

The second problem associated with the ${ }^{3} H_{4} \rightarrow{ }^{3} F_{4}$ transition is the possible nonradiative decay via the intermediate level ${ }^{3} \mathrm{H}_{5}$ which has a large probability especially in conventional silica fibre host. Therefore presently a majority of the TDFA modules is based on fluoride Tm-doped fibres with low-phonon energy, where the probability of nonradiative decay is lower. However, usage of fluoride fibres results in difficulties with fabrication and robustness of the host material. Furthermore, the fusion splicing to standard telecommunication fibre is impossible. This necessitates the use of butt mechanical splices that are comparatively lossy and prone to damage under high power pumping. These limitations have spurred the development of alternative host materials (Minelly and Ellison, 2002). Recently, gain of $10 \mathrm{~dB}$ (Ohara et al., 2002) and $12 \mathrm{~dB}$ (Cole and Dennis, 2001) were reported for a silica-based glass host. More than $20 \mathrm{~dB}$ gain was reached in a thuliumdoped silicate fibre (Samson et al., 2000), but this fibre still was not spliceable to standard silica-based optical fibres.

In spite of an extensive experimental effort in the field of the S-band TDFA's within the last ten years, there is a lack of detailed theoretical analysis of the amplifier performance by means of comprehensive numerical models that were successfully adopted in EDFA optimisation. The first numerical model of S-band TDFA was presented only in 2001 by Lee et al. Several other theoretical models of the thulium doped fibre have been presented before, dealing with multimode fluoride TDF for $800 \mathrm{~nm}$ band amplifier (Lincoln et al., 1991) and multimode silica TDF laser at $\approx 2 \mu \mathrm{m}$ (Jackson and King, 1999). An elaborated rateequation analysis of S-band TDFA in (Komukai et al., 1995) was used to show that the upconversion pumped TDF can be considered as a quasi-three level laser system and to derive an analytical expression for a local pump efficiency. However, neither the amplifier performance simulation, nor numerical model were presented there. In this paper we present a comprehensive, spectrally and spatially resolved numerical model of the TDFA. The aim of the modelling is to predict the performance of the S-band, silica-based TDFA. In contrary to the model referenced in (Lee et al., 2001), the silica fibre host and various pumping 
schemes are considered. Although two other S-band TDFA numerical models (Kasamatsu et al., 2002; Roy, 2002) appeared coincidentally with the presentation of our numerical model, both were dealing with fluoride fibre host. In the following paragraph we describe in detail our numerical model together with the fibre parameters that correspond as close as possible to the thulium-doped silica-based fibres prepared in the Laboratoire de Physique de la Matière Condensée (LPMC), Université de Nice. In the third paragraph we show application of the model to TDFA optimization and the TDFA performance simulation. The pumping scheme using single-wavelength around $1050 \mathrm{~nm}$, considered for the initial silica-based TDFA experiments, is treated in detail. The capability of the numerical model to describe the dual-wavelength scheme is then demonstrated on an example of 1410 and $1560 \mathrm{~nm}$ pumping.

\section{Numerical model}

The energy level diagram of the thulium with all transitions involved in the model is shown in Fig. 2. From the two conventions for energy level labeling the convention of dominating component of the mixed Russel-Saunders state is chosen. Particularly the first excited level is labeled ${ }^{3} F_{4}$ while the third one ${ }^{3} H_{4}$. More details about the labeling conventions can be found e.g. in the book of Caspary (2003). The levels ${ }^{3} F_{2}$ and ${ }^{3} F_{3}$ are very close and they are treated as single level (level No. 4). The stimulated absorption and emission rates $W_{i j}$ account for amplified spontaneous emission (ASE) in all relevant spectral bands (namely the $470 \mathrm{~nm}, 800 \mathrm{~nm}, 1470 \mathrm{~nm}$ and $1800 \mathrm{~nm}$ bands) and enable us to investigate each pumping scheme ever reported. The signal stimulated emission ${ }^{3} H_{4} \rightarrow{ }^{3} F_{4}$ is governed by $W_{31}$. Spontaneous decay processes are described by $A_{i j}^{r}$ and $A_{i}^{n r}$, the radiative and nonradiative decay rates, respectively. Since the nonradiative decay rates from ${ }^{3} F_{3}$ and ${ }^{3} H_{5}$ levels to the respective underlying level are high $\left(\gg 10^{5} \mathrm{~s}^{-1}\right)$, the atomic population density $N_{2}$ and $N_{4}$ of both levels are neglected in the model. The thulium concentration considered for the development of the thulium-doped silica fibre amplifiers is below $1000 \mathrm{ppm}$. For these levels of concentration the self-quenching process ${ }^{3} \mathrm{H}_{4},{ }^{3} \mathrm{H}_{6} \rightarrow$ ${ }^{3} F_{4},{ }^{3} F_{4}$ is not significant (Jackson and King, 1999) and this process is is neglected in the model. According to the energy level diagram one can write the rate equations for the population density in the relevant excited levels $N_{1}, N_{3}$ and $N_{5}$ as follows

$$
\begin{aligned}
\frac{d N_{1}}{d t} & =N_{0}\left(W_{01}+W_{02}\right)-N_{1}\left(W_{10}+W_{13}+W_{14}+A_{1}^{n r}+A_{10}^{r}\right) \\
& +N_{3}\left(W_{31}+W_{32}+A_{3}^{n r}+A_{32}^{r}+A_{31}^{r}\right)+N_{5}\left(A_{51}^{r}+A_{52}^{r}\right),
\end{aligned}
$$




$$
\begin{aligned}
\frac{d N_{3}}{d t}= & N_{0} W_{03}+N_{1}\left(W_{13}+W_{14}\right)+N_{5}\left(A_{5}^{n r}+A_{54}^{r}+A_{53}^{r}\right) \\
& -N_{3}\left(W_{35}+W_{32}+W_{31}+W_{30}+A_{3}^{n r}+\sum_{j=0}^{2} A_{3 j}^{r}\right), \\
\frac{d N_{5}}{d t}= & N_{0} W_{05}+N_{3} W_{35}-N_{5}\left(W_{50}+A_{5}^{n r}+\sum_{j=0}^{4} A_{5 j}^{r}\right),
\end{aligned}
$$

while it holds

$$
N_{t}=N_{0}+N_{1}+N_{3}+N_{5},
$$

where $N_{0}$ is the population density on ground level and $N_{t}$ is the average concentration of thulium in the core. For a measured rare-earth concentration profile $N(r)$ the $N_{t}$ is defined by $N_{t} \pi b^{2}=2 \pi \int_{0}^{\infty} N(r) r d r$, where $b$ is a doping radius, i.e. the half of the concentration profile FWHM. In general, the variables $N_{i}$ and $W_{i j}$ are functions of position $r, \varphi, z$ and time. Assuming that the thulium ions are excited homogeneously across the fibre cross section the expression for the transition rates $W_{i j}$, which describe the interaction of the electromagnetic field with the ions, can be written as

$$
W_{i j}(z)=\int_{0}^{\infty} \lambda \Gamma(\lambda) \sigma_{i j}(\lambda) \frac{\left(P_{\lambda}^{+}(z, \lambda)+P_{\lambda}^{-}(z, \lambda)\right)}{h c \pi b^{2}} d \lambda,
$$

where $\sigma_{i j}$ is the respective transition cross section, $h$ is Planck's constant, $c$ is the speed of light, $P_{\lambda}^{ \pm}$are the spectral power density of the radiation propagated in positive and negative direction of the fibre axis, respectively, and $\Gamma$ is the so-called overlap factor defined by

$$
\Gamma(\lambda)=\frac{\int_{0}^{\infty}|E(r, \varphi, \lambda)|^{2} N(r) r d r}{N_{t} \int_{0}^{\infty}|E(r, \varphi, \lambda)|^{2} r d r} .
$$

The transversal distribution of the electric field $E(r, \varphi)$ is calculated by iterative solution of the scalar wave equation, in which arbitrary rotationally symmetric refractive index profile is taken into account. At wavelengths below the cutoff it is assumed that all possible modes are equally excited. Typical curve of $\Gamma(\lambda)$ for a step-index fibre with parameters $a$ and $N A$ listed in Table I and step like concentration profile with $b=a$ is shown in Fig. 3a. The radial distribution of the intensity $|E(r)|^{2}$ for the guided modes at wavelengths of $1470 \mathrm{~nm}$ (signal), $1064 \mathrm{~nm}$ (pump) and $800 \mathrm{~nm}\left({ }^{3} F_{4} \rightarrow{ }^{3} H_{6}\right.$ ASE) is plotted in Fig. 3 b. Note that the fibre is single-mode for given parameters both at the S-band signal and at the $1064 \mathrm{~nm}$ pump while it supports two modes at ${ }^{3} F_{4} \rightarrow{ }^{3} H_{6}$ ASE. 
The radiation in the fibre can be viewed as a certain number of partial waves with the spectral width $\Delta \lambda_{k}$ around the wavelength $\lambda_{k}$ that have power $P_{k}$. This set of partial waves can describe the pump and signal radiation with a narrow spectral line, $\Delta \lambda_{k} \approx 0$, as well as a broadband ASE signal where $\Delta \lambda_{k}$ is given by the spectral width of the respective ASE band and by the number of points dividing this spectral interval. More specifically, the $\Delta \lambda_{k}$ of ASE partial waves is defined such that $\sum_{k} \sigma_{i j}\left(\lambda_{k}\right) \Delta \lambda_{k}=\int_{0}^{\infty} \sigma_{i j}(\lambda) d \lambda$, where the $\sigma_{i j}$ is the corresponding emission cross section. This concept of effective spontaneous emission bandwidths $\Delta \lambda_{k}$ allows us to switch easily between rather time consuming densely spectrally resolved calculation and faster approximative solution with lower number of dividing points, e.g. in the ASE bands other than the S-band.

In majority of comprehensive models of optical fibre amplifiers the propagation of the signals, the pumps and the ASE is governed by specific differential equation. In order to provide flexibility in choice of number of pumps and theirs wavelengths, the propagation of the each forward partial wave is described in this model by the same form of differential equation:

$$
\begin{aligned}
\frac{d P^{+}(\lambda)}{d z}= & \Gamma(\lambda) P^{+}(\lambda) \sum_{i j}^{\{10,30,31,50,32\}}\left(N_{i} \sigma_{i j}(\lambda)-N_{j} \sigma_{j i}(\lambda)\right) \\
& -\Gamma(\lambda) P^{+}(\lambda)\left(N_{0} \sigma_{02}(\lambda)+N_{1} \sigma_{14}(\lambda)+N_{3} \sigma_{35}(\lambda)\right) \\
& \left.+\Gamma(\lambda) \sum_{i j} 2 h 0,30,31,50,32\right\}
\end{aligned}
$$

Analogical equation holds for the backward partial waves $P^{-}(\lambda)$. The first term on the right hand side of equation (7) stands for the signal, pump or ASE stimulated absorption and emission in the respective transitions $i \leftrightarrow j$. Since no emission is present in the wavelength's region $\approx 1050 \mathrm{~nm}$, the second term describe uniquely the ground state absorption (GSA) and excited state absorption (ESA) at this band. The third term represents spontaneous generation of photons. Note that since a monochromatic input signal with $\Delta \nu \approx 0$ is assumed, this term vanish for signals. The background attenuation is expressed by coefficient $\alpha$ in the last term. The approach of only one form of propagation equation offers flexibility of choice of the pumping scheme at the expense of only slight increase of computational time. The propagation equations (7) for all partial waves, together with the set of the rate equations (1-4) under steady state conditions, are solved simultaneously along the fibre using Runge-Kutta-Gill method of the fourth order. 
Since the boundary conditions for the counterpropagating partial waves $P^{-}$of the ASE and pump are not known at the beginning of the fibre $(z=0)$, an effective iterative process (Karasek, 1997) must be applied.

The spectroscopic parameters used in the model were carefully chosen mostly from literature, some of them were estimated using JuddOfelt theory and several fluorescence lifetimes were measured on fibres made in LPMC. The Judd-Ofelt parameters $\Omega_{2}, \Omega_{4}$ and $\Omega_{6}$ are taken from (Zou and Izumitani, 1993). However, it should be noted that despite a great number of Judd-Ofelt spectroscopic studies of thulium in fluoride fibres, only a few works deal with silica host. The branching ratios $\beta_{i j}$, the probability of radiative transition from upper level $i$ to lower level $j$, were calculated using well known procedure of Judd-Ofelt analysis, described e.g. in (Amin et al., 1996) and (Spector et al., 1977). Zero background loss of the fibre is assumed throughout the paper. The transition cross sections are surveyed in Fig. 4 and other parameters are listed in Table I.

The cross sections were taken from (Jackson and King, 1999), except the transitions ${ }^{1} G_{4} \leftrightarrow{ }^{3} H_{6}$ and ${ }^{3} H_{4} \rightarrow{ }^{3} H_{6}$, whose transition strengths were estimated using Judd-Ofelt theory, and the transition ${ }^{3} H_{4} \leftrightarrow \leftrightarrow^{3} F_{4}$ (Guy et al., 2001). The transition cross sections in Guy et al. (2001) was measured in fluoride TDF, but it was checked by the Judd-Ofelt analysis that the transition strengths were consistent with those for silica. A maximum relative error of $14 \%$ was found, the error of which is usual in Judd-Ofelt calculations.

In order to validate the performance of the numerical model, a comparison with the model of fibre laser operating at $\lambda \approx 2 \mu \mathrm{m}$ (Jackson and King, 1999) was made and an excellent agreement of the calculated results has been found. The model of S-band TDFA itself was tested for the case of fluoride fibre host by comparison with experimental and theoretical results shown in Kasamatsu et al. (2002), however the results agreed only qualitatively. The discrepancies can be attributed most likely to the differences within the input parameters as some parameters were not stated in the referenced article.

\section{Results and discussion}

In Fig. 5a the spectral gain of 16 WDM channels in the S-band is presented for three values of fluorescence lifetime of the thulium energy level ${ }^{3} \mathrm{H}_{4}$ : the typical value in silica fibre host $\tau_{3}=14 \mu \mathrm{s}$ (Jackson and King, 1999), the value measured in TDF prepared in LPMC $\tau_{3}=45 \mu \mathrm{s}$ and finally the record value measured in the silicate fibre truly compatible with standard optical fibres $\tau_{3}=55 \mu$ s (Cole and Dennis, 2001). 
Table I. Parameters used in the numerical simulations.

\begin{tabular}{|c|c|c|c|c|}
\hline Parameter & Unit & Symbol & Value & Remark \\
\hline Tm concentration & $m^{-3}$ & $N_{t}$ & $1.56 \times 10^{25}$ & \\
\hline Core diameter & $\mu \mathrm{m}$ & $2 a$ & 2.6 & \\
\hline Numerical aperture & & $N A$ & 0.3 & \\
\hline $\mathrm{LP}_{11}$ cutoff & $\mathrm{nm}$ & $\lambda_{c o}$ & $1018 \mathrm{~nm}$ & \\
\hline Fluorescence & $\mu \mathrm{s}$ & $\tau_{5}$ & 784 & Jackson, 1999 \\
\hline \multirow[t]{2}{*}{ lifetimes } & $\mu \mathrm{s}$ & $\tau_{3}$ & 45 & Measured. \\
\hline & $\mu \mathrm{s}$ & $\tau_{1}$ & 430 & Measured. \\
\hline \multirow[t]{3}{*}{ Radiative lifetimes } & $\mu \mathrm{S}$ & $1 / \sum_{i=0}^{4} A_{5 i}$ & 860 & Digonnet, 1993 \\
\hline & $\mu \mathrm{s}$ & $1 / \sum_{i=0}^{2} A_{3 i}$ & 650 & Digonnet, 1993 \\
\hline & $\mu \mathrm{s}$ & $1 / \overline{A_{10}}$ & 3500 & Digonnet, 1993 \\
\hline Judd-Ofelt & $m^{2}$ & $\Omega_{2}$ & $3.26 \times 10^{-24}$ & Zou and \\
\hline \multirow[t]{2}{*}{ parameters } & $m^{2}$ & $\Omega_{4}$ & $1.20 \times 10^{-24}$ & Izumitani, 1993. \\
\hline & $m^{2}$ & $\Omega_{6}$ & $0.46 \times 10^{-24}$ & \\
\hline \multirow[t]{8}{*}{ branching ratios } & & $\beta_{54}$ & 0.03 & Estimated by Judd- \\
\hline & & $\beta_{53}$ & 0.11 & Ofelt analysis; \\
\hline & & $\beta_{52}$ & 0.30 & Amin et al., 1996; \\
\hline & & $\beta_{51}$ & 0.06 & Zou and \\
\hline & & $\beta_{50}$ & 0.50 & Izumitani, 1993; \\
\hline & & $\beta_{32}$ & 0.03 & Spector et al., 1977. \\
\hline & & $\beta_{31}$ & 0.09 & \\
\hline & & $\beta_{30}$ & 0.88 & \\
\hline
\end{tabular}

Codirectional pump of $1000 \mathrm{~mW}$ at $1064 \mathrm{~nm}$ and input power of $10 \mu \mathrm{W}$ of each signal channel was assumed. For each value of $\tau_{3}$ the optimum fibre length was found. Since the pumping scheme exploiting single wavelength $\approx 1050 \mathrm{~nm}$ is considered as the first candidate for the silica-based TDFA demonstration (thanks to availability of the high-power pump source and less complexity than the dual-wavelength schemes) several optimization issues of TDFA under this arrangement are addressed in the following. In the Fig. 5b we can see the effect of fibre waveguide parameters on the single pass gain. In analogy with the EDFA, the gain increase with increasing the numerical apperture of the fibre and narrowing the core diameter. The power $1000 \mathrm{~mW}$ of the forward pump at $1064 \mathrm{~nm}$ and one $10 \mu \mathrm{W}$ signal channel at $1470 \mathrm{~nm}$ were considered. Length of the fibre was $5 \mathrm{~m}$. The signal gain is deteriorated by the ASE, dominantly the one in the $800 \mathrm{~nm}$ band. Introducing an artificial filter of the ASE in this band would lead to 
substantial amelioration of the gain, as represented by the dashed lines in Fig. 5b. E.g. for the $N A=0.4$, the difference between gain maxima is $10 \mathrm{~dB}$.

Optimization of the fibre length and pump wavelength is shown in Fig. 6. According to the Fig. 6a, an estimation of the optimum fibre length is given by the position along the fibre where the gain has maximum. Lower increase of gain in the beginning of the fibre for pump powers $1000 \mathrm{~mW}$ and $1500 \mathrm{~mW}$ is caused by strong ASE, mainly in the 800 and $470 \mathrm{~nm}$ bands. For comparison, dashed lines represent the calculated gain when the ASE is suppressed. In Fig. 6b a single pass gain is plotted versus pump wavelength. For higher levels of input pump power the optimum pump wavelength shifts towards shorter wavelength, similarly as in the case of the fluoride TDFA (Lee et al., 2001). With decreasing wavelength the GSA becomes lower and it results in more uniform distribution of the population inversion along the fibre. Integral value of the population inversion over entire fibre length is higher than in case of higher GSA. This strong argument for choosing shorter pump wavelength rather than the wavelength $1064 \mathrm{~nm}$, the one of the Nd:YAG lasers and some Yb:fibre lasers, can be moderated in case of bidirectional pumping, with the same total power. In $2000 \mathrm{~mW}$ total pump, for example, the difference of the maximum gain and the gain for $1064 \mathrm{~nm}$ pump wavelength is $11 \mathrm{~dB}$ for codirectional pumping in contrary to only $5.7 \mathrm{~dB}$ for bidirectional arrangement. Slight increase of maximum gain can be achieved with bidirectional pumping.

In analogy to the flouride fibre host it is expected that using an optimized dual-wavelength pumping will lead to enhancement of power conversion efficiency of the silica-based TDFA. Therefore the numerical model was developed to allow for treatment of arbitrary pumping scheme. However the complexity of optimization issues of dualwavelength pumping schemes is doubled with respect to the singlewavelength scheme. Such an optimization study is behind the scope of this article and will be the subject of future work. An example of application of the developed numerical model to study the dualwavelength pumping scheme (1410 and $1560 \mathrm{~nm}$, see Fig. 1, Kasamatsu et al., 2000) of the TDFA is presented in Fig. 7. The additional pump at $1560 \mathrm{~nm}$ can enhance the gain but for high $1560 \mathrm{~nm}$ pump powers the ASE in the $2 \mu \mathrm{m}$ band significantly deteriorate the S-band signal gain. Therefore an optimum pump power combination exists as can be seen from Fig. 7b. The amplifier gain is less sensitive to $1560 \mathrm{~nm}$ pump power level for the arrangement where the $1560 \mathrm{~nm}$ pump propagates counterdirectionally with respect to the signal. 


\section{Conclusions}

This paper has described in detail the developed comprehensive numerical model of TDFA, taking into account the silica fibre host. The model is spectrally and spatially resolved and is general in terms of pumping schemes and the fibre type (e.g. silica and fluoride fibre). The model was also used for predicting the S-band amplifier performance with dual wavelength pumping and for optimisation of the TDFA parameters: core radius and numerical apperture, fibre length, pump wavelength and pump power. The simulations are helpful in obtaining physical insight on various processes in the TDFA, particularly it enables to assess quantitatively the influence of the ASE in different bands on the amplifier performance. The results of the modelling show that above $20 \mathrm{~dB}$ gain can be achieved with the core material composition developed in the LPMC. However high pump power of 2 Watts in $1050 \mathrm{~nm}$ pump band is necessary to obtain such a gain. Further improvement of quantum efficiency of the $1470 \mathrm{~nm}$ transition has to be achieved in order to make the silica based TDFA attractive comparing to its competitors within the S-band optical amplifiers, namely the fluoride-fibre based TDFA and the Raman amplifiers.

\section{Acknowledgements}

The authors would like to thank David Simpson from Victoria University in Melbourne, Australia, for the measurements of the ${ }^{3} F_{4}$ level fluorescence lifetime and Prof. Anderson S. L. Gomes from Universidade de Pernambuco, Brazil for sharing his experience in advanced TDFA pumping methods. This work was partially supported by the "Ministère de la Jeunesse, de l'Education Nationale et de la Recherche", France, contract No. 224/2002 and by the Grant Agency of the Czech Republic, contract No. 102/02/D168.

\section{References}

Amin, J., B. Dussardier, T. Schweizer, and M. Hempstead: 1996, "Spectroscopic analysis of Er transitions in lithium niobate". J. of Luminescence 69, 17-26.

Antipenko, B. M., A. A. Mak, O. B. Raba, K. B. Seiranyan, and T. V. Uvarova: 1983, "New laser transmition in the Tm ion". Sov. J. of Quantum Electron. 13(4), 558-560.

Caspary, R.: 2003, Applied rare-earth spectroscopy for fiber laser optimization. Shaker, Aachen.

Cole, B. and M. L. Dennis: 2001, "S-band amplification in a thulium doped silicate fiber". In: OFC'01, Anaheim, USA; p. TuQ3. 
Digonnet, M. J. F.: 1993, Rare earth doped fiber lasers and amplifiers. New York, Marcel Dekker.

Guy, S., W. Meffre, A. M. Jurdyc, B. Jacquier, F. Roy, P. Baniel, D. Bayart, A. L. Sauze, C. Collet, and J. J. Girard: 2001, "Spectroscopic studies of Tm doped glasses for S-band amplifiers". In: In Tech. Digest of OAA'01, Stresa, Italy, July 1-4, paper OWB5.

Jackson, S. D. and T. A. King: 1999, "Theoretical modeling of Tm-doped silica fiber lasers". J. Lightwave Technol. 17(5), 948-956.

Karasek, M.: 1997, "Optimum design of Er-Yb codoped fibers for large-signal highpump-power applications". J. Quantum Electronics 33(10), 1699-1705.

Kasamatsu, T., Y. Yano, and T. Ono: 2000, "Laser-diode pumping (1.4 and 1.56 $\mu \mathrm{m}$ ) of gain-shifted thulium-doped fibre amplifier". El. Lett. 36(19), 1607-1609.

Kasamatsu, T., Y. Yano, and T. Ono: 2002, "1.49- $\mu$ m-band gain-shifted thuliumdoped fiber amplifier for WDM transmission systems". J. of Lightwave Technol. 20(10), 1826-1837.

Komukai, T., T. Yamamoto, T. Sugawa, and Y. Miyajima: 1995, "Upconversion pumped thulium-doped fluoride fiber amplifier and laser operating at $1.47 \mu \mathrm{m}$ ". IEEE J. Quantum Electron. 31(11), 1880-1888.

Lee, W. J., B. Min, J. Park, and N. Park: 2001, "Study on the pumping wavelength dependency of S+band fluoride based thulium doped fiber amplifiers ". In: OFC-2001 Technical-Digest, Anaheim, Vol. 2. pp. TuQ 5-1-4.

Lincoln, J. R., W. S. Brocklesby, F. Cusso, J. E. Townsend, A. C. Tropper, and A. Pearson: 1991, "Time resolved and site selective spectroscopy of thulium doped into germano- and alumino-silicate optical fibres and preforms". J. of Luminescence 50, 297-308.

Minelly, J. and A. Ellison: 2002, "Applications of antimony-silicate glasses for fiber optic amplifiers". Optical Fiber Technology 8(2), 123-138.

Ohara, S., N. Sugimoto, Y. Kondo, K. Ochiai, Y. Kuroiwa, Y. Fukasawa, T. Hirose, $\mathrm{H}$. Hayashi, and $\mathrm{S}$. Tanabe: $2002, " \mathrm{Bi}_{2} \mathrm{O}_{3}$-based glass for S-band amplification". In: Proc of SPIE, Vol. 4645. pp. 8-15.

Roy, F.: 2002, L'amplificateur à fibre dopée thulium. Ph.D. thesis, L'Université de Franche-Comté.

Samson, B. N., N. J. Traynor, D. T. Walton, A. J. G. Ellison, J. D. Minelly, J. P. Trentelman, and J. E. Dickinson: 2000, "Thulium doped silicate fiber amplifier at 1460-1520 nm". In: Optical Amplifiers and Their Applications, 9-12, Quebec, Canada, Vol. 44. pp. 247-249.

Spector, N., R. Reisfeld, and L. Boehm: 1977, "Eigenstates and radiative transition probabilities for $\mathrm{Tm}^{3+}\left(4 f^{12}\right)$ in phosphate and tellurite glasses". Chem. Phys. Lett. 49(1), 49-53.

Zou, X. and T. Izumitani: 1993, "Fluorescence mechanisms and dynamics of Tm singly doped and $\mathrm{Yb}, \mathrm{Tm}$ doubly doped glasses". J. of Non-Cystalline Solids $162,58-67$. 


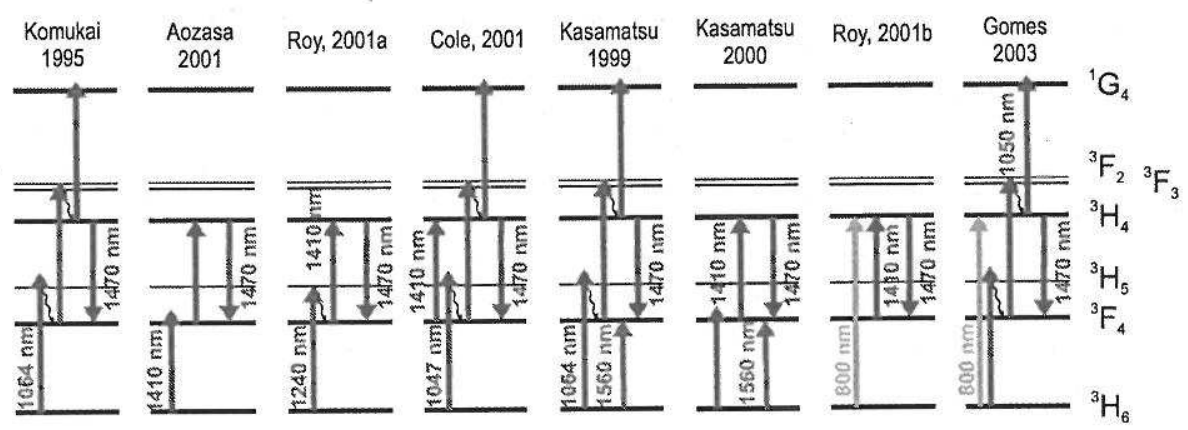

Figure 1. Upconversion pumping schemes for TDFA that have been reported so far.

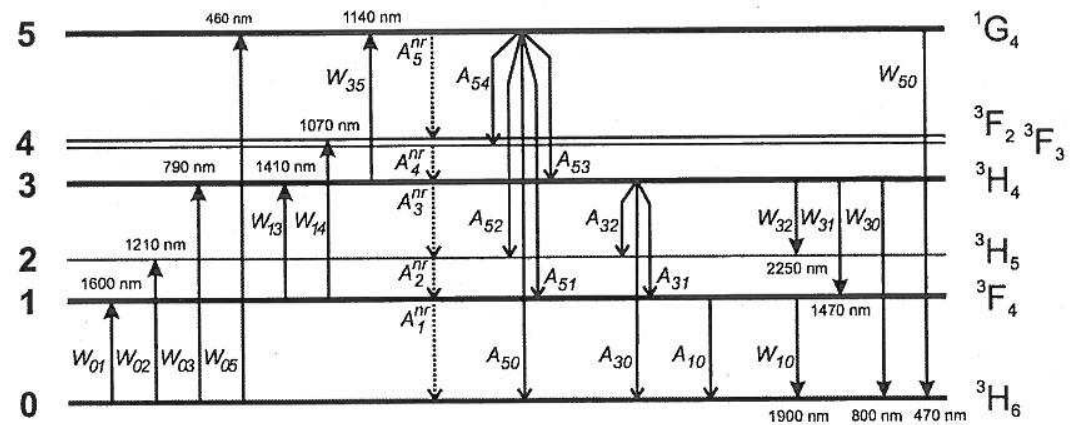

Figure 2. Tm energy level diagram with all transitions involved in the model. 


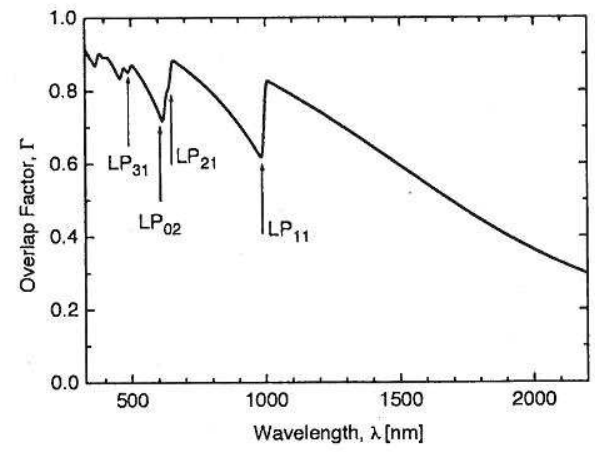

(a)

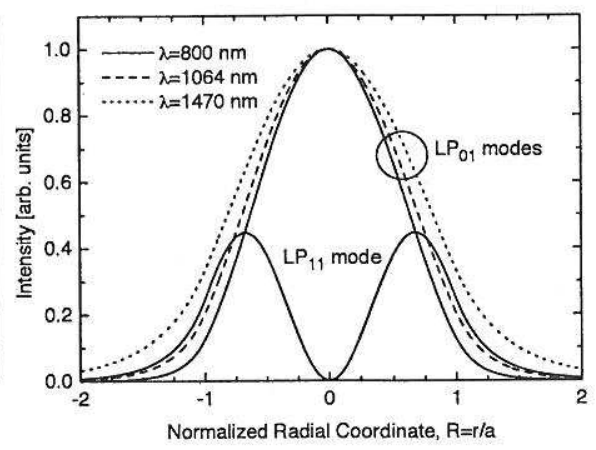

(b)

Figure 3. (a) Spectral dependence of the overlap factor $\Gamma(\lambda)$ for a step-index fibre with parameters $2 a=2.6 \mu \mathrm{m}, N A=0.3$ and $b=a$. The cutoff wavelengths of the first four higher order modes are noted in the figure. (b) The radial distribution of the intensity $|E(r)|^{2}$ for the guided modes at wavelengths of the ${ }^{3} F_{4} \rightarrow{ }^{3} H_{6}$ ASE, the pump wavelength of $1064 \mathrm{~nm}$ and the S-band signal.

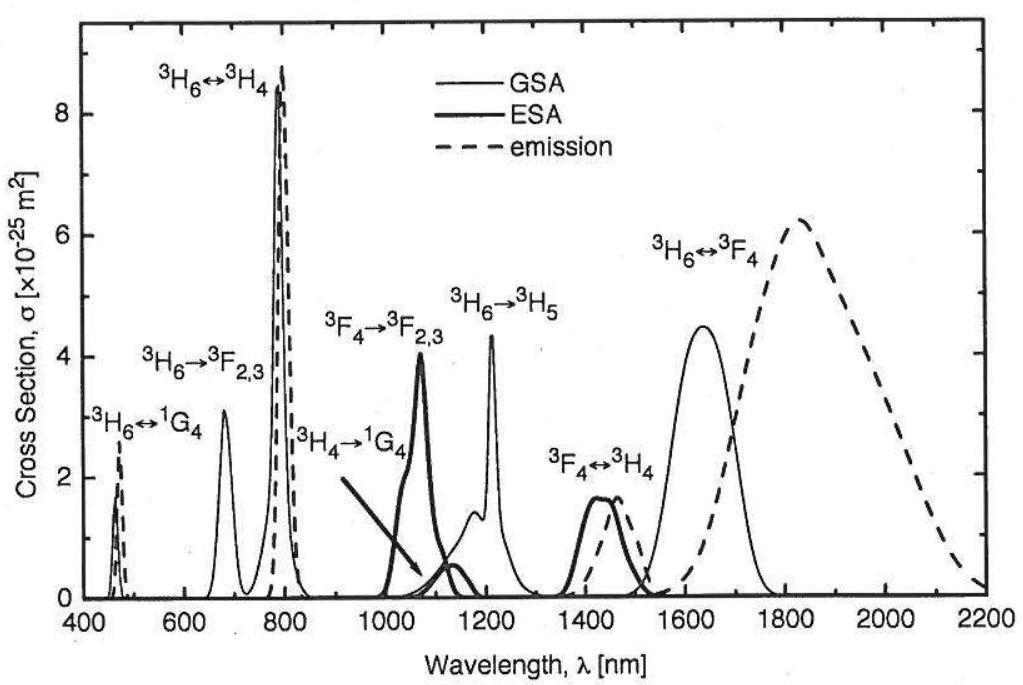

Figure 4. Absorption and emission cross section spectra of thulium. 


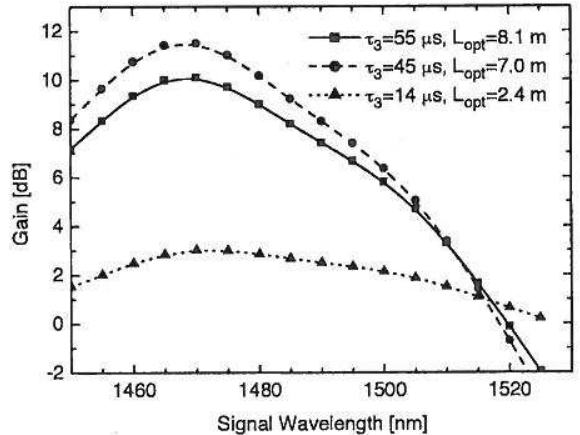

(a)

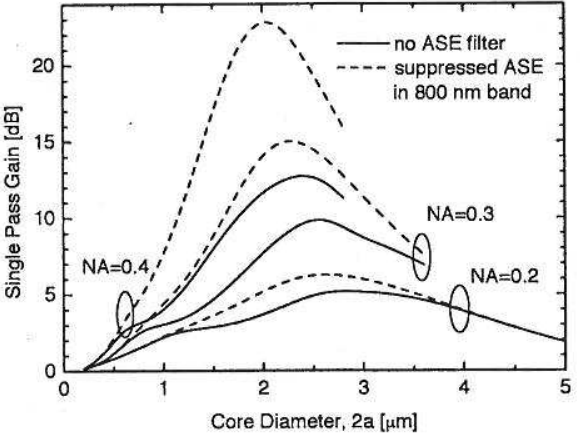

(b)

Figure 5. (a) Spectral gain of $16 \mathrm{WDM}$ channels in the S-band for three values of the fluorescence lifetime $\tau_{3}$. (b) Single pass gain vs. core radius for several values of $N A$. Only the core radii for which the fibre is single-mode in the S-band are taken into account. 


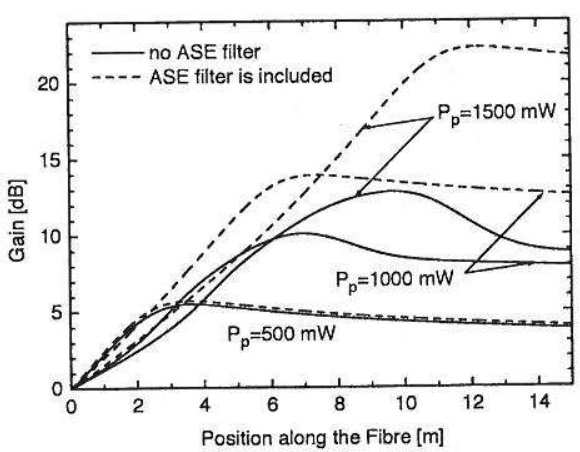

(a)

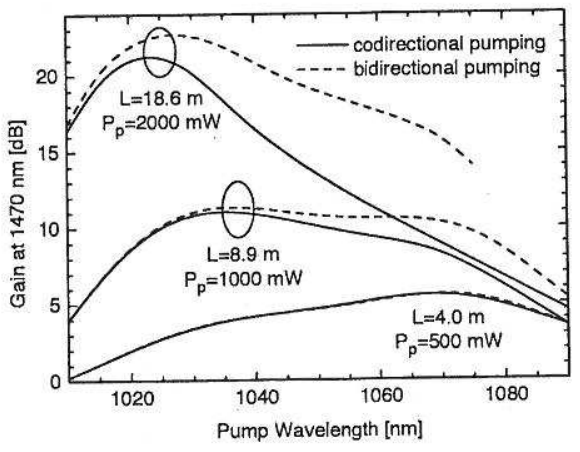

(b)

Figure 6. (a) Optimization of the fibre length. The signal gain along the TDF is calculated for several input pump powers at $1064 \mathrm{~nm}$. Dashed lines are calculated excluding the effect of ASE at $800 \mathrm{~nm}$ band. (b) Optimization of the pump wavelength. Solid line is for codirectional pumping while dashed lines stand for bidirectional pumping scheme. No ASE filter was included. Total input pump power and the length of the fibre are shown under the curves.

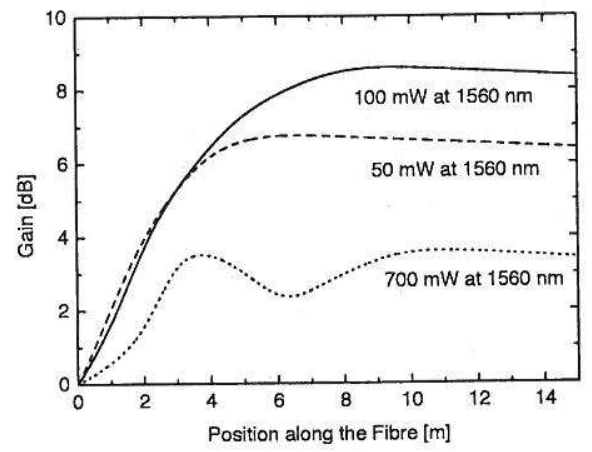

(a)

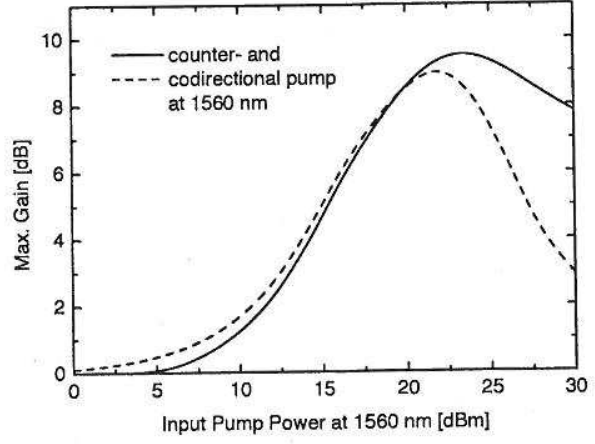

(b)

Figure 7. (a) Gain along the TDF when the amplifier is pumped codirectionally by two wavelengths at $1410 \mathrm{~nm}$ and $1560 \mathrm{~nm}$ according to the pumping scheme in Fig. 1, Kasamatsu et al., 2000. The pump power at $1410 \mathrm{~nm}$ was always $1000 \mathrm{~mW}$. (b) Maximal gain along the fibre with respect to the input power at $1560 \mathrm{~nm}$ for both counter- and codirectional $1560 \mathrm{~nm}$ pump arrangement. Fibre length were $15 \mathrm{~m}$. 